d'un jour de jeûne, chaque homme apporte dans le plus grand de ses champs du pain de blé et du lait ainsi que quelques baguettes de woulkeffa (Dombeya bruceana) dont l'écorce est enlevée sur place. Ayant fait une fois le tour complet du terrain (sens inverse des aiguilles d'une montre), le propriétaire plante une baguette dans le sol, puis une autre au milieu du champ et une troisième diamétralement opposée à la première. Revenu au centre, il jette du lait autour de lui en le prenant dans le creux de la main et en imitant le geste du semeur. Il fait de même avec du pain émietté, puis il consomme le reste des aliments en priant Dieu de préserver la récolte des oiseaux, des singes, des maladies des céréales et de la guerre.

Le beurre est employé dans les recettes les plus diverses. Au Bégamder, il entre, avec le Tupa rhyncopetalum, dans un remède contre l'avortement. On soigne la fièvre avee du beurre pur, l'orchite avec du beurre bien égoutté. Il entre dans la composition d'onguents ou de régimes pour la dysurie, les douleurs de reins, la syphilis, les vers intestinaux, la stérilité. Il entre également dans des recettes pour s'échapper de prison, pour acquérir de l'argent, pour être doué de voyance (1).

A l'occasion de sacrifices offerts pour des raisons magico-religieuses, l'officiant en oint les cheveux des assistants en même temps qu'il leur fait partager la communion.

Les chrétiens n'en consomment pas, non plus que du lait, pendant les nombreux jours de jeûne. Durant le carême, les gens d'église et ceux qui communient évitent même de s'oindre la tête, le beurre pénétrant dans l'intérieur de corps par les racines des cheveux (2).

\title{
UNE RACE DES ÉTATS DU LEVANT SOUS MANDAT FRANÇAIS : LA RACE BOVINE BÉLÉdIE
}

\author{
par
}

\section{R. FERRANDO}

Chef de travaux à l'Ecole nationale vétérinaire de Lyon.

Parmi les variétés, souvent inattendues, d'animaux de l'espèce bovine qui existent au Levant, on arrive à distinguer une race qui paraît excellente : la race Bélédie. Bonne laitière et rustique à la fois, elle mérite d'être sérieusement étudiée. Parfaitement adaptée au milieu où elle évolue elle gagnerait à être soigneusement sélectionnée. Son élevage prenant ensuite un grand développement elle

(I) Cf. M. Griaule : Le Livre de Recettes d'un Dabtara, voir au mot beurre, à l'index des remèdes.

(2) Cette dernière croyance est rapportée par d'A ввAdIE, Manuscrit 265, folio 20, Bibliothèque Nationale. 
pourrait être exportée : ce serait alors une source de profit pour ce pays.

En approfondissant l'étude de l'aire géographique, de la morphologie, des qualités de cette race, nous pourrons envisager les méthodes à mettre en œuvre pour l'améliorer et pour en augmenter les débouchés.

\section{Aire géographique.}

On rencontre cette race dans les régions riches de la Syrie et du Liban. Son nom de Bélédie lui vient de l'arabe "bled" : pays, campagne, désignant ainsi l'animal indigène. Son berceau semble être les environs de Damas. Tout autour de cette ville il existe une région très riche : la Ghouta. Arbres, prairies, ruisseaux ; lorsque par Dmeir on arrive de Palmyre, le contraste est grand entre cette

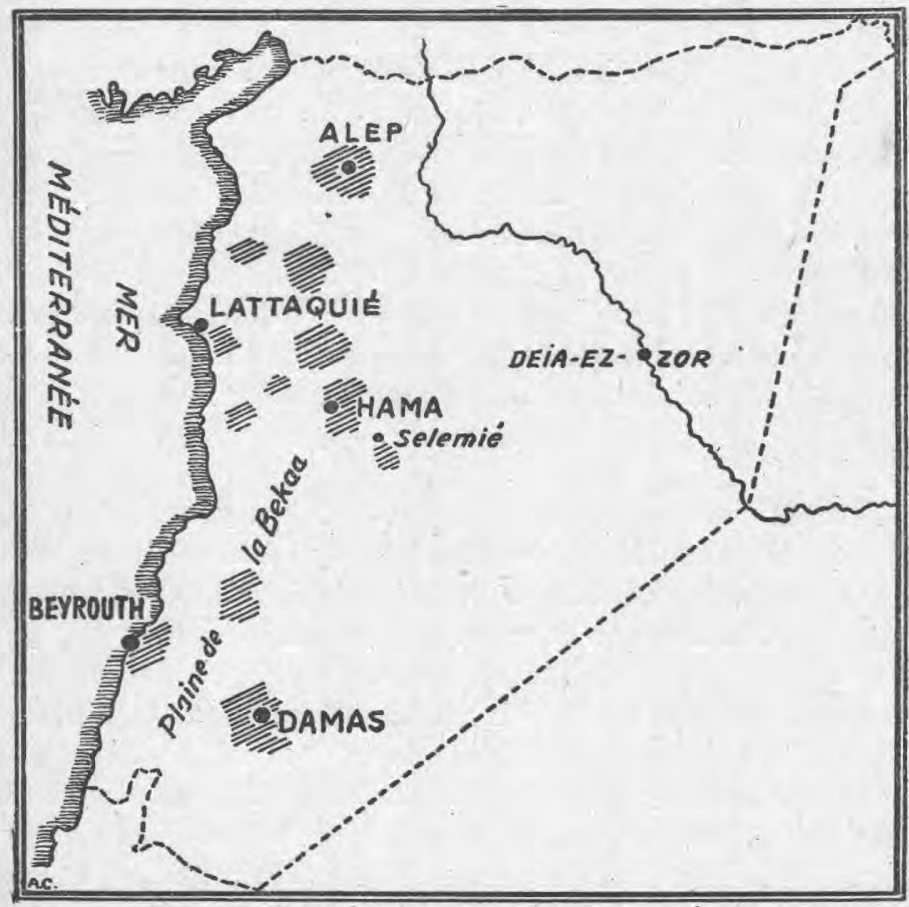

WCENTRES D'ÉLEVAGE DELARACE BÉLÉDIE

richesse et les plaines arides qu'on vient de quitter. Irriguée par la rivière Barada qui prend sa source dans l'Anti-Liban, la Ghouta, région plate, à sol d'alluvions, a une superficie d'environ 30.000 hectares. Cette oasis, entièrement labourable, est d'une fertilité étonnante. On y trouve, entre autres productions, des prairies naturelles 
et artificielles. Le climat doux et humide permet à cette végétation de se développer avec une grande vigueur. Pendant la quarantaine de kilomètres que dure la traversée de cette région on se croirait transporté dans quelque riche province de France.

Sous les arbres, au milieu des prairies, les vaches pâturent. La douceur du climat permet de les y laisser presque constamment.

De là, il semble que la race ait rayonné dans tout le Levant. On la trouve plus particulièrement dans les régions à production agricole développée : région de Beyrouth, plaine de la Bekaa (surtout vers Chtaura et Zahlé), environs de Homs, de Hama, dans les Alaouites, environs d'Alep, à Deir-ez-Zor même. Si elle prospère d'abord là où le sol est fertile et les prairies abondantes, on peut dire aussi qu'elle s'adapte parfaitement bien dans les régions pauvres, où elle conserve ses belles qualités, malgré la rudesse des conditions de vie. L'aire géographique de la race bélédie est done très étendue. Divers croisements ont eu lieu avec des races voisines ou locales moins intéressantes et difficilement définissables qui ont eneore étendu l'aire géographique de la race. Notons enfin que la grande diversité des milieux entraîne des variations' du format.

En résumé, on trouve la vache Bélédie plus spécialement aux environs de Damas qui peuvent être considérés comme son berceau, au Liban, dans la Békaa, les Alaouites, les alentours d'Alep.

S'adaptant bien elle gagnerait à être protégée et sélectionnée plus sérieusement. L'étude de sa morphologie et de ses aptitudes ne fera que nous confirmer dans cette impression.

\section{Morphologie.}

La race est médioligne et eumétrique. Un examen approfondi permet de noter un mélange de caractères qui font hésiter à la classer parmi les subconcaves. Il est vraisemblable que des croisements multiples sont intervenus dans sa formation.

Cette région de l'Asie fut un lieu de passage; des croisements se sont fatalement produits. La vache Bélédie peut très bien avoir dans ses ancêtres des représentants de la race asiatique des steppes (bos, taurus asiaticus) qui est rectiligne. En effet, on trouve parfois chez elle des cornes en coupe ou en lyre haute. Mais on trouve également, et le plus souvent, des eornes en couronne ou en crochet, parfois même des cornes très atrophiées indiquant un ancêtre concave et même peut-être un ancêtre sans cornes. Ajoutons à cela le ehignon parfois saillant et la queue en crosse et nous pouvons encore songer à des ancêtres eonvexes. Le bos taurus Scythicus ne serait-il pas allé jusqu'en Asie mineure? D'autre part, MM. GAILLARD et le docteur PORTET, dans "La Faune momifiée de l'ancienne Egypte ", indiquent et prouvent qu'une race bovine sans cornes 
vécut autrefois sur les bords du Nil. Des migrations de peuples, des échanges multiples s'effectuèrent entre l'Egypte et la. Syrie. Ce dernier pays fut toujours un carrefour où les peuples et les raees animales se sont sans arrêt affrontés et mêlés. Pourquoi donc les animaux de l'espèce bovine n'en auraient-ils pas fait autant. Certes ce sont là de simples hypothèses; cependant, en ce qui concerne la race Bélédite, l'étude de sa morphologie dévoile son origine métisse.

On est surtout frappé de la très grande finesse des sujets de cette race. La vache Bélédie (fig. 1) rappelle la vache Jerseyaise. Comme elle, e'est un peu la gazelle de l'espèce. Le milieu, le climat, l'aptitude sont causes de cet affinement qui se rencontre même chez le taureau.

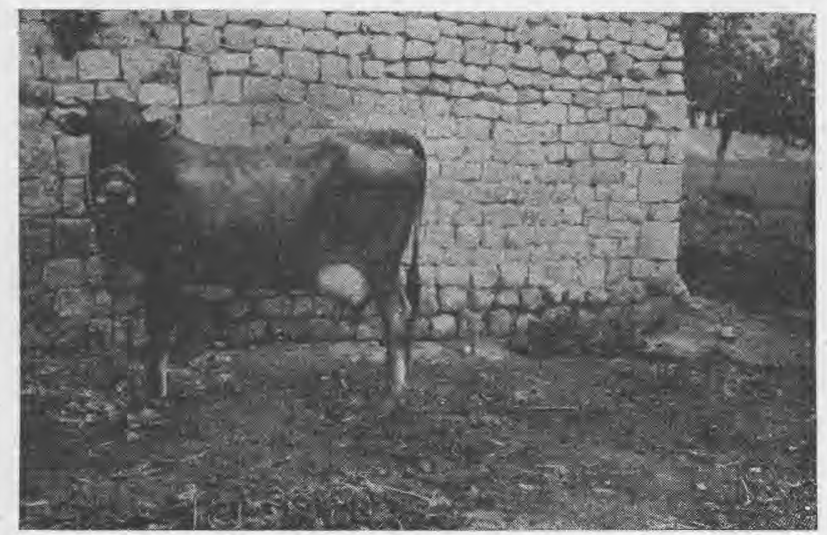

Fig. 1. - Vache Bélédie. Alep.

La tête est fine et courte, portant une dépression frontale légèrement accusée. Le profil est subconcave. Le mufle est moyen très légèrement retroussé.

Les cornes sont en général insérées sur la ligne du chignon. Le plus souvent elles sont ramenées en couronne ou en crochets. On trouve aussi des cornes en coupes ou en lyre haute et fréquemment des cornes atrophiées, petites, rabougries, comme rognées ; parfois il n'y a qu'une corne. Cette grande variété de cornage nous a fait hésiter pour classer cette race selon la méthode de Baron.

L'encolure est svelte chez la vache, très musclée chez le taureau et le bœuf. Le mâle a un garrot large et haut (fig. 2). Le train antérieur est excessivement développé.

Le fanon est abondant.

La poitrine est large, ample, profonde, bien descendue.

Le dessus est droit. Les hanches sont assez saillantes et larges comme tout le bassin. La queue est longue et fine. Son attache, quel- 
quefois en cimier, est le plus souvent horizontale. La fesse est droite, la cuisse plate et maigre. Les membres solides, très fins, très nerveux, ont d'excellents aplombs.

La peau est mince, souple et mobile; les poils sont courts et brillants.

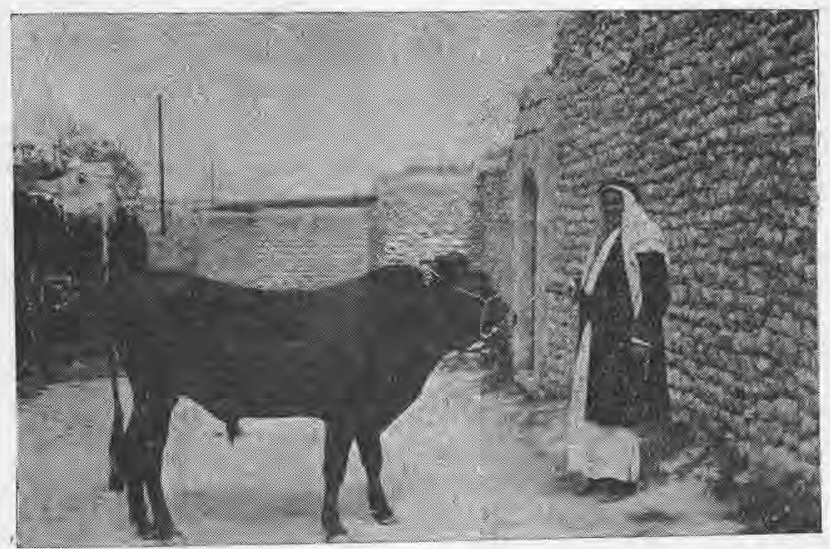

Fig. 2. - Taureau Bélédie. Alep.

Les caractères laitiers sont très développés. La mamelle est volumineuse, bien conformée, très irriguée. Malheureusement, certaines pratiques, telles que l'introduction de hampes de plumes dans les trayons, provoquent des mammites. La conséquence de ces affections dues à ces manœuvres maladroites est qu'on rencontre couramment des vaches ayant un ou deux quartiers atrophiés. Lorsque de tels accidents ne se sont pas produits, on peut dire que la mamelle répond à toutes les données classiques. On peut reprocher à certains sujets la petitesse de leurs trayons, ce qui n'enlève rien à leurs qualités laitières.

Taille : 1 mètre 45 à 1 mètre 55 chez le mâle.

1 mètre 35 à 1 mètre 40 chez la femelle.

Poids : 600 à 700 kilos chez le mâle.

400 à 500 kilos chez la femelle.

La race est brune. Le mufle, le dessous de la langue, les paupières, le pourtour de l'anus et de la vulve sont pigmentés. Chez le mâle il existe une cupule remontant assez haut. Les cornes, blanches à Ia base, ont les extrémités noires. Si ces caractères de pigmentation sont généraux et constants, la robe varie quelque peu du fauve claire au brun foncé. 
MENSURATIONS

\begin{tabular}{|c|c|c|c|c|c|c|c|c|c|c|c|c|c|}
\hline & $\begin{array}{l}\text { Tau- } \\
\text { reau } \\
4 \text { ans }\end{array}$ & $\begin{array}{l}\text { Tau- } \\
\text { reau } \\
9 \text { ans }\end{array}$ & $\begin{array}{l}\text { Vache } \\
5 \text { ans }\end{array}$ & $\begin{array}{l}\text { Vache } \\
5 \text { ans }\end{array}$ & $\begin{array}{l}\text { Vache } \\
3 \text { ans }\end{array}$ & $\begin{array}{l}\text { Vache } \\
5 \text { ans }\end{array}$ & $\begin{array}{l}\text { Vache } \\
5 \text { ans }\end{array}$ & $\begin{array}{l}\text { Tau- } \\
\text { reau } \\
4 \text { ans }\end{array}$ & $\begin{array}{l}\text { Vache } \\
5 \text { ans }\end{array}$ & $\begin{array}{l}\text { Vache } \\
6 \text { ans }\end{array}$ & $\begin{array}{l}\text { Vache } \\
9 \text { ans }\end{array}$ & $\begin{array}{l}\text { Vache } \\
6 \text { ans }\end{array}$ & $\begin{array}{l}\text { Tau- } \\
\text { reau } \\
7 \text { ans }\end{array}$ \\
\hline Taille ............... & 1,51 & 1,56 & 1,39 & 1,43 & 1,42 & 1,33 & 1,44 & 1,55 & 1,32 & 1,30 & 1,32 & 1,38 & 1,47 \\
\hline Tour de canon .......... & 0,19 & 0,22 & 0,165 & 0,17 & 0,18 & 0,17 & 0,17 & 0,19 & 0,17 & 0,16 & 0,17 & 0,17 & 0,19 \\
\hline Tour droit de poitrine ... & 1,80 & 2,05 & 1,67 & 1,80 & 1,81 & 1,72 & 1,80 & 1,90 & 1,78 & 1,79 & 1,86 & 1,85 & 1,85 \\
\hline Tour biais de poitrine .... & 1,95 & 2,15 & 1,80 & 1,90 & 2,00 & 1,85 & 1,90 & 2,05 & 1,88 & 1,90 & 1,98 & 1,95 & 2,05 \\
\hline Distance orbitaire ....... & 0,235 & 0,29 & 0,19 & 0,19 & 0,19 & 0,18 & 0,21 & 0,29 & 0,19 & 0,20 & 0,21 & 0,20 & 0,23 \\
\hline Largeur des hanches .... & 0,47 & 0,50 & 0,44 & 0,49 & 0,50 & 0,50 & 0,52 & 0,51 & 0,46 & 0,49 & 0,51 & 0,51 & 0,50 \\
\hline
\end{tabular}


Dans un premier cas, la robe fauve claire ressemble à celle de la race Tarentaise, avec des lunettes et une bordure de poils noirs autour du mufle. Les extrémités, les bracelets de la couronne et le toupillon de la queue sont noirs. Les parties postérieures des canons (ligne des tendons) sont recouverts de poils gris argent qui forment transition entre la muqueuse du mufle et sa bordure noire. Le mâle est toujours plus foncé. Il possède des lunettes plus accentuées. On trouve chez lui des charbonnures sur le cou, les épaules et le fanon. Les poils du fourreau sont noirs.

- Dans un second cas, la robe, qui est fauve foncée tirant légèrement sur le brun fauve, possède les mêmes particularités que la précédente. noire.

Enfin, quelques individus possèdent une robe brune, presque

- Dans tous les cas les mamelles sont toujours pigmentées.

On préfère la première robe. La deuxième est moins prisée, et les sujets qui possèdent la dernière sont peu recherchés et gardés comme animaux de travail.

\section{Aptitudes.}

La race, dont toute la conformation révèle la finesse, est bonne laitière; on peut même dire excellente laitière, si on considère le poids de la vache Bélédie et le milieu où elle vit.

Les contrôles laitiers qui ont été eftectués viennent démontrer que cette aptitude est une réalité et non une légende bâtie sur des déclarations d'éleveurs plus ou moins dignes de foi.

La produetion est de 3.000 à 3.500 kilogrammes de lait par lactation. En considérant la courte durée de cette dernière : 195 à 300 jours ( 245 jours en moyenne), on voit que la quantité journalière est assez forte. Des vaches d'élite arrivent à produire, durant les deux premiers mois de leur lactation, 15,18 et même 23 kilogrammes de lait par jour. Ce lait est assez riche en matière grasse ; il en faut 22 à 25 litres pour.faire un kilogramme de beurre.

Les courbes de contrôle laitier que nous présentons ici, (graphiques I, II, III) et qui ne proviennent pas de vaches remarquablement conformées, sont cependant intéressantes à examiner. Leur étude et les divers renseignements que nous avons pu obtenir, nous amènent aux conclusions suivantes :

La vache Bélédie est une excellente laitière. Sa période de lactation est relativement courte. On note pendant la lactation quelques chutes de débit dues à l'alimentation irrégulière. Enfin, la sécrétion semble parfois se tarir en quelques semaines.

Mais, pour pouvoir se prononcer définitivement sur l'allure de la. 
lactation, il faudrait examiner de nombreuses courbes et pouvoir également contrôler les belles vaches que nous avons très souvent rencontrées. Pour cela le temps et les moyens nous manquèrent. Cependant, dès à présent, nous nous permettons d'affirmer l'existence

Graphique I.

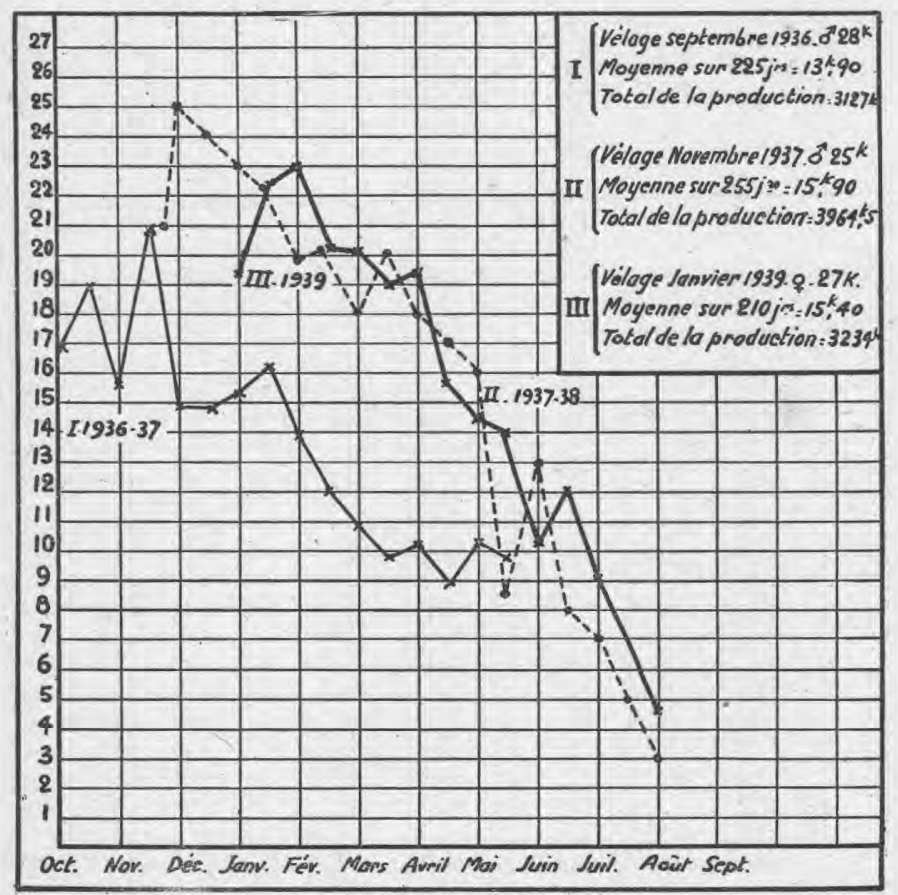

Vache. "KARIMÉE" née en 1930

de ces qualités laitières qui sont, en outre, susceptibles d'amélioration.

De caractère très doux, les taureaux et les bœufs sont d'excellents animaux de travail. Leur train antérieur très développé, leurs membres solides permettent un effort régulier et soutenu. A maintes reprises, nous en avons vu qui, attelés à la charrue du pays nommée "edda", labouraient, sans fatigue apparente, des champs parfois très escarpés. Les vaches ne travaillent pas; elles ne sont élevées que pour la production laitière.

Les qualités précédentes et l'absence totale de précocité, font de ces sujets de très médiocres animaux de boucherie. Leur rendement varie de 45 à $48 \%$ et leur viande, dure, n'est pas d'un goût très agréable. D'ailleurs, Syriens et Libanais ne consomment guère que du mouton et la colonie française fait un large usage de viande frigorifiée de provenance étrangère. 


\section{Elevage.}

Bien que chaque propriétaire tienne à ses vaches, leur exploitation ne donne pas lieu à de grands soins. Leur vie se passe presque constamment au grand air et dans les pâturages, quand il y en a. Malheureusement, ils sont limités à quelques régions en dehors desquelles ils ne durent que de la fin janvier à la fin mai.

En été, les animaux ne reçoivent souvent qu'un peu de cette paille de créréales finement pulvérisée et appelée teben ; on y mêle

Graphique II.

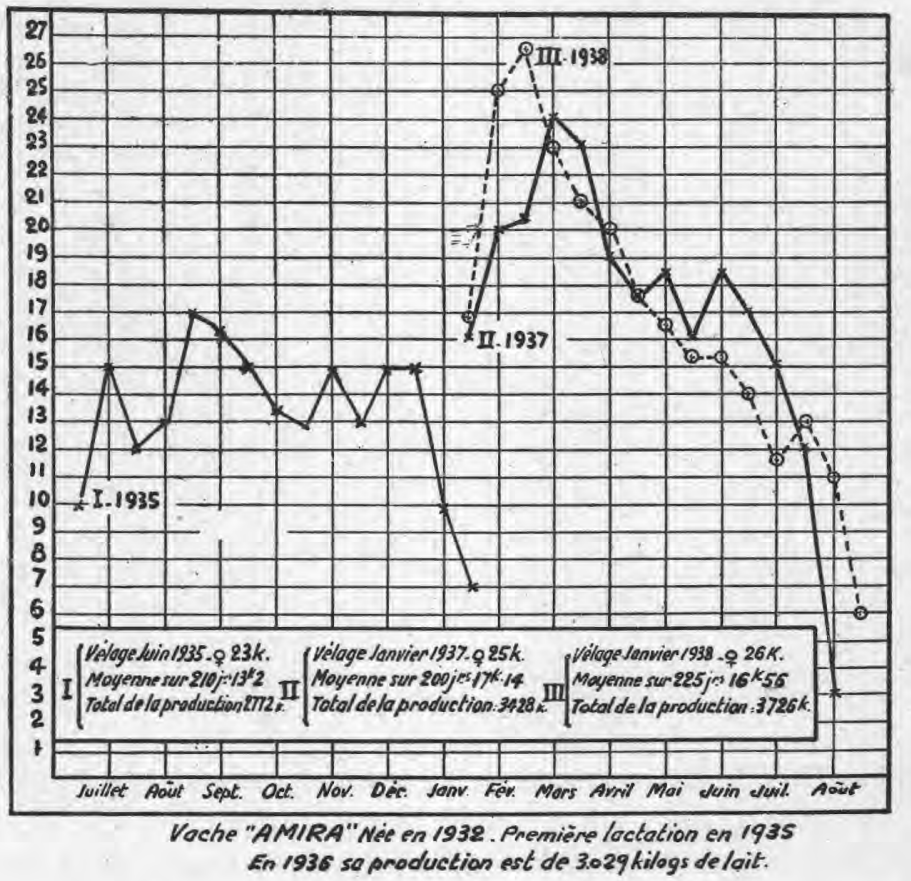

du son, des excréments de vers à soie, un peu d'orge ou de farine d'orge. Aux environs des villes, le soir venu, les animaux sont rentrés à l'étable ou, le plus souvent, dans un local qui n'en a que très vaguement l'aspect. Pourtant, malgré ces soins et cette alimentation rudimentaires, la vache produit et produit bien.

Des taureaux connus, utilisés longtemps et ne servant qu'un cercle déterminé de vaches, toujours le même, font la saillie. Ceux qui ne sont pas destinés à la reproduction sont castrés à un an et utilisés par la suite comme animaux de travail. Les génisses, vendues d'après la réputation laitière de leur mère, sont saillies pour la première fois entre deux ans et deux ans et demi, en mai ou en juin. 
Ainsi le vêlage a lieu au début du printemps suivant, à l'époque de la pousse de l'herbe. Les vaches conservées pendant plusieurs années sont vendues, le cas échéant, d'après leur production laitière. Leur prix n'excède jamais 2.000 à 2.500 francs (1939).

Graphique III.

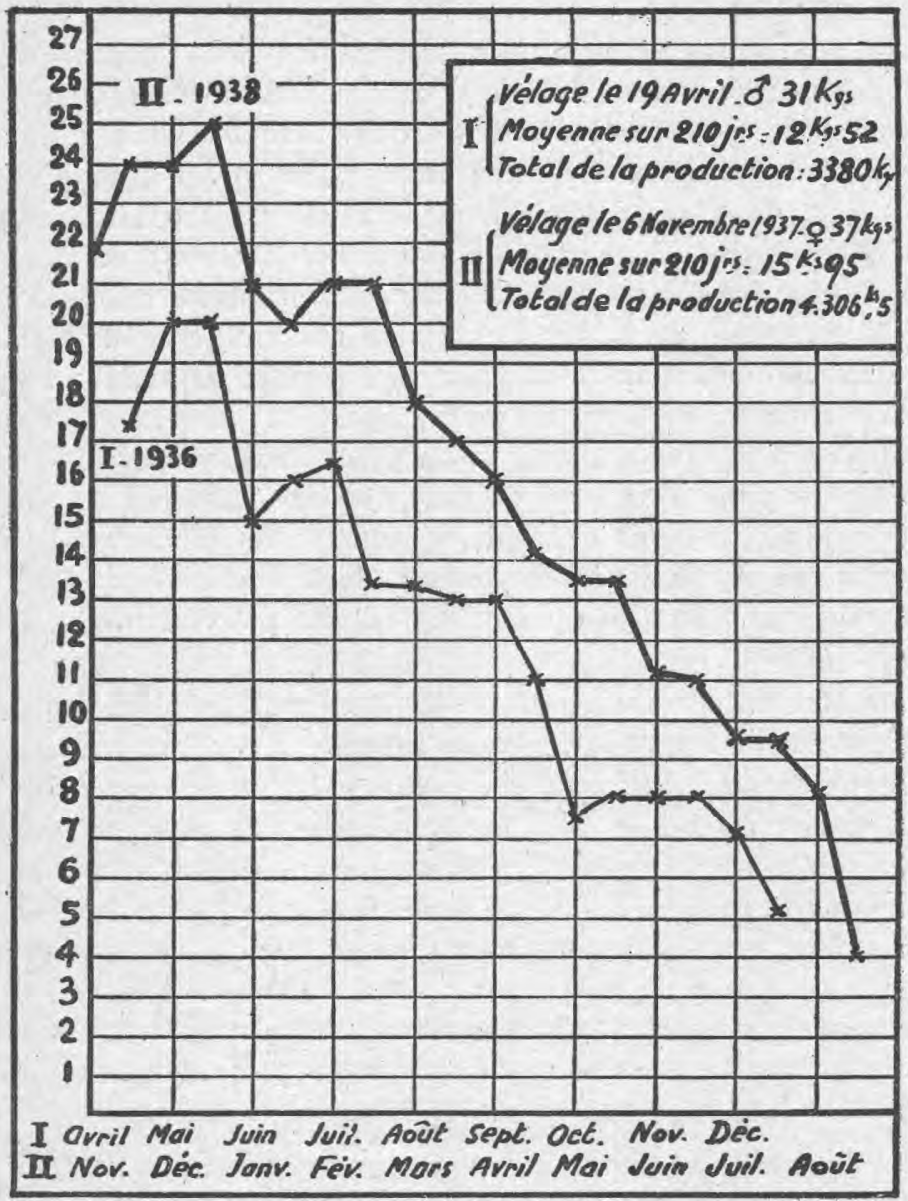

Voche BLACK-BEAUTY" "née en 1927

Chaque propriétaire vend son lait dans des conditions d'hygiène souvent déplorable. Cependant, nous avons visité à Alep une laiterie-beurrerie qui est un modèle d'organisation et de propreté. Elle possède onze centres de ramassage et d'écrémage, pasteurise la crème et produit un beurre excellent. Il faut déplorer que cette installation soit unique et que son exiguité ne lui permette pas une 
grosse fabrication. De tels établissements sont à généraliser et à encourager.

Les aptitudes du bétail, certains procédés d'élevage et certaines conditions économiques, incitent à penser qu'il serait facile d'améliorer considérablement la race et son exploitation.

\section{A mélioration.}

Si on consomme beaucoup de laitage en Orient : lait en nature, leben (lait caillé), beurre, fromages divers, il faut remarquer qu'une des sources de cette production se trouve être les états du Levant sous mandat français. De nombreux pays voisins, la Palestine notamment, désireux d'avoir chez eux des vaches laitières, sont venus acheter dans la Ghouta quantité d'excellents sujets. On a même dû prendre des mesures sévères pour empêcher cette exportation qui menaçait de priver le pays de ses meilleurs animaux. Ce grave inconvénient n'existerait plus si l'élevage de la race recevait une forte impulsion. A ce moment, étant en possession de sujets plus a méliorés et plus nombreux, on pourrait rétablir l'exportation vers les pays cités plus haut et vers d'autres encore plus éloignés. L'Afrique du Nord par exemple, qui manque de vaches laitières, trouverait au Levant des animaux déjà endurcis aux climats chauds, résistant aux piroplasmoses (1) et pouvant donc s'acclimater facilement.

Pour arriver à cela, il faut toute une organisation dont nous allon, essayer de tracer les grandes lignes.

Il convient, avant tout, d'améliorer la nourriture. Dans les régions riches où vit le plus habituellement la race, ce problème est très facile et ne consiste pas tant à renforcer la production agricole que la ration elle-même. Ici il faut agir plutôt sur l'éleveur que sur le sol, car les régions riches, dont nous parlons plus haut, n'ont guère besoin de développer leurs cultures. Ailleurs, il faut laisser la steppe désertique aux moutons : son irrigation, sa mise en valeur est une œuvre de longue haleine; parfois même, elle est impossible.

On doit toujours tenir compte des possibilités des pays et des régions et parallèlement ne pas oublier que, dans l'évolution de l'élevage, le mouton commence, la vache laitière termine. Vouloir tout bouleverser, tout changer d'un seul coup, c'est aller à l'encontre de lois de la nature et de l'économie rurale. Il faut laisser la vache

(1) Le vétérinaire capitaine L. Prgoury a identifié au Levant Babesiella berbera, Anaplasma marginale, Theileira mutans et Piroplasma bigeminum. De ces observations cliniques il ressort que l'infection semble peu grave : quelques accès aigus de gravité moyenne, le plus souvent des symptômes assez vagues : légère hyperthermie, inappétence, anémie. La guérison survient sans traitement en deux ou trois semaines. 
là où elle peut vivre facilement. Il faut persuader simplement son éleveur d'augmenter la quantité et la qualité de la ration en Iui faisant entrevoir la possibilité de débouchés, donc de bénéfices et de profits. Il pourra alors entreprendre de lui-même, ou sur les conseils des dirigeants, certaines cultures, en particulier celle des racines fourragères. Par endroit, elles permettront de remédier à la pénurie des fourrages. Mais il faut pour cela que le paysan comprenne qu'indirectement, par la vente du lait, de telles entreprises lui rapporteront. Les concours agricoles, les vacheries, le crédit agricole, les laiteries-beurreries coopératives doivent faciliter sa tâche et l'aider à saisir la nécessité et l'importance de l'amélioration du bétail.

Au début, il faut organiser dans les principaux centres d'élevage des concours agricoles qui seraient annuels ou bisannuels et auraient lieu à Damas, Homs, Hama, Alep, Lattaquié, accessoirement à Deir-ez-Zor, au moment des fêtes de printemps, bien que cette région soit secondaire au point de vue de l'élevage des animaux de l'espèce bovine. Naturellement, il est nécessaire de faire comprendre à l'éleveur le but du concours agricole. Il y parviendra vite en s'apercevant que les primes vont aux meilleurs sujets et qu'il a intérêt à présenter de nombreux et beaux individus. Avec un minimum de réclame ces réunions deviendront populaires et le nombre des participants augmentera, certainement, de plus en plus.

Simples au début, on pourra peu à peu renforcer les épreuves au fur et à mesure que l'éleveur comprendra l'idée du concours. A ce moment-là, c'est-à-dire deux ou trois ans après les débuts, il sera possible de juger les animaux d'après une échelle de points telle que celle-ci :

\begin{tabular}{|c|c|c|}
\hline & Vaches & Taureaux \\
\hline Conformation $\ldots \ldots \ldots \ldots \ldots \ldots \ldots \ldots$ & 3 & 4 \\
\hline 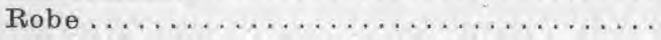 & 1 & 1,5 \\
\hline Pigmentation $\ldots \ldots \ldots \ldots \ldots \ldots \ldots \ldots$ & 1 & 1,5 \\
\hline Finesse $\ldots \ldots \ldots \ldots \ldots \ldots \ldots \ldots \ldots \ldots \ldots \ldots \ldots \ldots \ldots \ldots \ldots$ & 1 & 3 \\
\hline 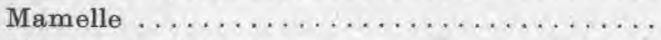 & 3 & \\
\hline Signes empiriques $\ldots \ldots \ldots \ldots \ldots \ldots \ldots$ & 1 & \\
\hline & $-\frac{}{10}$ & $\overline{10}$ \\
\hline
\end{tabular}

Dès cet instant la sélection pourra commencer. On suivra alors par la suite les sujets d'élite et leurs produits. Cette particularité de l'élevage local, d'après laquelle seul un petit nombre de taureaux font saillie, permettra de survieiller facilement des génitèurs et leurs produits. On marquera aussi lors des concours les belles individualités. Le seul fait de les représenter l'année suivante entraînera l'octroi d'une sorte de prime de conservation plus forte pour le taureau. 
On interdira temporairement la vente à la boucherie et à l'étranger des sujets ainsi marqués.

Nous ne parlons pas d'organiser le contrôle laitier. Pour l'instant cela est impossible et, bien que les génisses soient vendues d'après la réputation laitière de leurs mères, on ne peut, par suite de la dispersion des vaches, faire un contrôle sérieux dont l'établissement. donnerait naissance aux multiples truquages et trafics chers à l'Orient. Ils en fausseraient totalement les résultats et le but. C'est seulement dans les concours et au bout de quelques années qu'il sera possible d'inaugurer des épreuves laitières et beurrières.

Deux organismes sont également à eréer : les vacheries et les laiteries-beurreries coopératives.

10 Les vacheries, dont les éleveurs verront les animaux dans les concours, seront des exemples placés sous les yeux de ces derniers. Leurs taureaux serviront de géniteurs. Les étrangers pourront y étudier la race et ses possibilités. Enfin, les techniciens y feront d'intéressantes observations dont bénéficieront les paysans.

$2^{\circ}$ La création de crédits agricoles et de laiteries-beurreries coopératives peut influencer favorablement le développement de la race. Elles délivreront l'éleveur de la contrainte des usuriers et des accapareurs. Plus libre, assuré du lendemain, il pourra mieux perfectionner son bétail.

Au Levant, les cités vivent en parasites de la campagne et la maintiennent dans une étroite dépendance. L'enchevêtrement du commerce et du crédit agricole est, dans ces pays, une des causes de la pauvreté des populations rurales. En effet, à l'occasion de sa venue à la ville, le producteur entre en, relation avec le commerçant en produits agricoles. Celui-ci a tôt fait de se transformer en créancier, car le paysan imprévoyant, qui dépense plus qu'il ne gagne, a nécessairement besoin d'argent. Et pour cette cause, le propriétaire est obligé de céder ses récoltes à vil prix. Aussi, par crainte d'être exploité, il préfère les vendre sur, place. Il gagne moins, mais il n'a pas la sensation d'être volé par le commerçant rapace du souk. Ce sont done des intermédiaires qui viennent acheter dans les villages et la campagne. Ils pratiquent des cours souvent inattendus qui varient avec chaque endroit : "l'économie rurale en est fragmentée et instable, la liaison entre les régions étant toujours le fait de la spéculation plutôt que d'un trafic régulier. " La création d'un crédit agricole et d'organismes coopératifs viendra supprimer ces inconvénients. Producteurs et consommateurs y gagneront, l'hygiène du lait en sera favorablement affectée, une industrie laitière prendra naissance. De tels organismes existent à l'état embryonnaire à Alep et fonctionnent parfaitement; on ne peut donc objecter l'impossibilité. 
IMPORTATIONS EN PRODUITS DE LAITERIE EN SYRIE ET AU LIBAN

\begin{tabular}{|c|c|c|c|c|}
\hline $\begin{array}{c}\text { Pays expéditeurs } \\
\text { Lait en bloes, en poudre } \\
\text { ou condensés, sucré ou non. } \\
\text { En kilogramme net }\end{array}$ & 1935 & 1936 & 1937 & 1938 \\
\hline France $\ldots \ldots \ldots \ldots \ldots \ldots$ & 31.128 & 65,486 & 60.306 & 84.519 \\
\hline $\begin{array}{l}\text { Allemagne } \ldots \ldots \ldots \ldots \ldots \ldots \ldots \ldots \ldots \ldots \\
\text { Alle } \ldots \ldots \ldots \ldots \ldots \ldots\end{array}$ & 348 & 150 & - & 34.019 \\
\hline Autriche $\ldots \ldots \ldots \ldots \ldots \ldots$ & - & 50 & - & - \\
\hline Belgique $\ldots \ldots \ldots \ldots \ldots \ldots \ldots$ & 11.166 & 9.883 & 5.697 & 900 \\
\hline Egypte ............... & 500 & 21 & 6 & 16 \\
\hline Etats-Unis . . . . . . . . . . & 2.939 & 6.696 & 7.568 & 9.074 \\
\hline Grande-Bretagne .......... & 12.508 & 10.616 & 10.681 & 5.321 \\
\hline Pays-Bas ............. & 90.383 & 67.865 & 105.357 & 54.406 \\
\hline Suisse . . . . . . . . . . . . & 39.816 & 33.276 & 41.643 & 77.136 \\
\hline Autres pays .............. & 8.617 & 7.734 & 23.641 & 10.084 \\
\hline Kilogrammes nets....... & 197.405 & 201.777 & 254.599 & 241.456 \\
\hline Beurre frais ou salé (1) & & & & \\
\hline France $\ldots \ldots \ldots \ldots \ldots \ldots \ldots$ & 50 & 338 & 1.663 & 2.423 \\
\hline Egypte ................ & 1.605 & 18 & 2.000 & - \\
\hline Etats-Unis . . . . . . . . . . & 6 & 200 & - & - \\
\hline Grande-Bretagne $\ldots \ldots \ldots \ldots$ & 2.038 & 310 & 1.136 & 11 \\
\hline Irak $\ldots \ldots \ldots \ldots \ldots \ldots \ldots$ & 11.888 & 100 & - & - \\
\hline Italie $\ldots \ldots \ldots \ldots \ldots \ldots \ldots \ldots$ & 387 & 9 & - & - \\
\hline Palestine................ & 379 & 30 & 一 & 23 \\
\hline Pays-Bas ................ & 5.783 & 19.291 & 20.487 & 11.208 \\
\hline Roumanie.............. & $\longrightarrow$ & 1.576 & - & - \\
\hline Suède $\ldots \ldots \ldots \ldots \ldots \ldots \ldots \ldots$ & - & 320 & - & - \\
\hline Suisse $\ldots \ldots \ldots \ldots \ldots \ldots \ldots$ & 6.200 & 3.000 & - & - \\
\hline Autres pays . . . . . . . . . . & 100.979 & 44.528 & 21.516 & 12.741 \\
\hline Kilogrammes nets....... & 129.315 & 69.720 & 46.802 & 26.406 \\
\hline
\end{tabular}

(1) Pour 1935, y compris le beurre fondu et celui dénommé "Samnels» dans l'Orient.

Le fait que la race vive dans des régions bien déterminées et assez peu étendues permet également d'envisager cette création. Au moment du ramassage du lait, on pourra même donner aux éleveurs conseils et encouragements, leur indiquer la date des prochains concours, leur parler des avantages multiples qu'ils peuvent retirer du développement de leur élevage, effectuer une surveillance sanitaire, vacciner et distribuẹ des médicaments. Certes, tout cela 
n'ira pas sans difficultés. Au début, il faudra faire comprendre à l'éleveur, au consommateur, mais surtout aux pouvoirs publics, l'intérêt que peut représenter pour eux tous et pour le pays le développement de cet élevage et de l'industrie laitière. Ce n'est qu'après bien des années d'efforts et de persuasion, que des possibilités d'exportation seront ouvertes. La réputation de la race, déjà grande, mais augmentée encore par les résultats acquis, le permettra facilement.

Dès ce moment, l'éleveur, ayant saisi tout l'intérêt qu'il a d'écouter les conseils et les directives venues du service de l'élevage, et voyant que tout fonctionne dans le seul but de l'aider et de l'enrichir, s'appliquera à faire mieux. La sélection pourra alors être intensifiée, mais, sous peine d'inefficacité, elle devra toujours avoir pour base les concours et les primes qui, par exemple, pourraient provenir d'une partie des bénéfices des laiteries-beurreries coopératives. Parallèlement à cela, il faut appliquer dès maintenant certaines mesures générales qui sont :

10 Défense sanitaire.

$2^{\circ}$ L'interdiction d'importer d'autres races. Les essais effectués avec les animaux des races hollandaises et jerseyaises n'ont pas donné de résultats positifs. On se demande, d'ailleurs, pourquoi on a voulu tenter d'acclimater en Syrie des races aussi perfectionnées, qui demandent à vivre sur des pâturages abondants et sous des climats doux. Il ne faut pas croiser la race Bélédie qui, le jour où elle sera nourrie et sélectionnée correctement, doit avoir une lactation plus longue, une production plus forte. On aura alors au Levant une très belle et très bonne race laitière bien adaptée au milieu naturel.

30 La castration et l'utilisation comme animaux de trait des taureaux qui ne sont ni de race pure, ni de bonne conformation. Une petite prime pourrait avoir raison des propriétaires hésitants.

Ce sont là quelques mesures d'ordre général; il serait nécessaire, le cas échéant, de les codifier en mettant au point une organisation détaillée. La mise en ouvre de ce plan pourrait, à notre humble avis, donner des résultats appréciables.

L'amélioration d'une race est, surtout dans ces pays, une œuvre de longue haleine. La lutte pour une telle entreprise sera rude. On se heurtera à de faux principes, à des sinécures établies de longue date, à l'appétit de lucre de commerçants et d'usuriers tout puissants qui ne se laisseront pas enlever, sans réagir, une grande source de profits : l'exploitation du paysan. Il faudra aussi combattre cette paresse terrible, à qui on ne peut opposer que l'appât du gain. A force de patience, de travail tenace, de stricte discipline et avec 
quelques moyens financiers, il est possible de venir à bout de cette entreprise. Les résultats atteints paieront largement, au sens propre comme au sens figuré, de tous ces efforts.

Dès à présent, il est nécessaire de songer à cette période d'après guerre où il faudra redonner une vive impulsion à l'organisation agricole de notre empire. Toutes nos richesses, même les moindres, devront être exploitées. Dans cette intention, nous avons pensé que le développement d'une petite race locale pourrait contribuer à cette ouvre tout en apportant aux états du Levant sous mandat français un peu d'hygiène et de bien-être.

Beyrouth, le 4 juin 1940.

Lyon, le 28 janvier 1941.

\title{
BIBLIOGRAPHIE
}

J. EDdé. Géographie de la Syrie et du Liban.

Latron. La vie rurale en Syrie et au Liban.

L. Pigoury. Les piroplasmoses bovines au Levant.

Dr Portet et Gamland. La faune momifiée de l'ancienne Egypte.

M. Saba. L'Inspection des viandes et des abattoirs on Syrie. Thèse, Alfort, 1936.

Sanson. Traité de Zootechnie.

Statistiques des douanes du port de Beyrouth.

\section{PRÉPARATION ET PROPRIÉTÉS DES ESTERS LACTIQUES}

\author{
par G. GÉNIN
}

Ingénieur chimiste E. P. C.

Au cours des diverses études que nous avons publiées ici même sur les applications des produits contenus dans le sérum du lait, nous avons été amenés à parler assez fréquemment des esters lactiques et nous avons insisté en particulier sur quelques fabrications à la base desquelles on peut utiliser ces esters. WHITtien et Rogers [1], OLIve [2] et Burton [3] ont également signalél'emploi de ces esters et ils ont décrit leur préparation en partant d'acide lactique fabriqué par fermentation du sérum. Cet acide, en effet, peut être transformé en différents mono et diesters dont certains présentent un intérêt assuré. Plus récemment, L. T. SмiтH et H. V. Claborn [4] ont également étudié, au Bureau of Dairy Industry du Ministère de l'Agriculture des Etats-Unis, la préparation d'esters lactiques déjà connus et de certains nouveaux, en utilisant directement le lactate de calcium ou le lactate de sodium bruts que l'on obtient par la fermentation du sérum. A certains points de vue, 